\author{
${ }^{1 *}$ A.A. Eleuov, ${ }^{2}$ N.N. Tungatarov, ${ }^{3}$ F.K. Yakhiyayev \\ ${ }^{1,2}$ Faculty of Mechanic-Mathematical, Al-Farabi Kazakh National University, Almaty, Kazakhstan; \\ ${ }^{3}$ Scientific-Research Institute of Mathematics and Mechanics, \\ Al-Farabi Kazakh National University, Almaty, Kazakhstan \\ ee-mail: Eleuov@mail.ru, Nourmat@mail.ru
}

\title{
A numerucal numerical method for the restoration of the five diagonal symmetric matrices from the spectral data
}

\begin{abstract}
In this work the possibility of restoration of real symmetrical five diagonal final matrixes using four numerically sequences is studied. Three from these four numerically sequences are interpreted as sets of eigenvalues of the considered matrix and else of two matrixes, obtained from considered matrix deleting some diagonal elements. The concrete formulas of construction of matrix elements using four sets of eigenvalues are obtained.
\end{abstract}

Key words: differential equations, band matrix, spectral problems, eigenvalues, five-diagonal symmetric matrix.

\section{Introduction}

Strip matrixes appear from the discreteness of boundary-value problems for the linear differential equations in the section. The algorithm of the restoration of three diagonal matrices from two spectra can be found in the work [1]. Inverse spectral problems for five diagonal unitary matrices are examined in the work [2]. In this work the algorithm of the single-valued restoration of five diagonal symmetrical matrix is indicated. At the beginning of work the properties of the eigenvalues of the symmetrical five diagonal matrixes are investigated

$$
A=\left[\begin{array}{cccccccc}
a_{0} & b_{0} & c_{0} & & & & & \\
b_{0} & a_{1} & b_{1} & c_{1} & & & & \\
c_{0} & b_{1} & a_{2} & b_{2} & c_{2} & & & \\
& c_{1} & b_{2} & a_{3} & b_{3} & c_{3} & & \\
& & \ddots & \ddots & \ddots & \ddots & \ddots & \\
& & & c_{n-4} & b_{n-3} & a_{n-2} & b_{n-2} & c_{n-2} \\
& & & & c_{n-3} & b_{n-2} & a_{n-1} & b_{n-1} \\
& & & & & c_{n-2} & b_{n-1} & a_{n}
\end{array}\right]
$$

All numbers $a_{0}, a_{1}, \ldots ., a_{n}, b_{0}, b_{1}, \ldots ., b_{n}$ are real, and numbers $c_{0}, c_{1}, \ldots ., c_{n-2}$ are positive.

\section{Main body}

From the work [3] it is known that the eigenvalues of matrix $A$ are real. This is obvious. In our work we prove the not entirely obvious properties of eigenvalues of matrix $A$. Denote $P_{n+1}(\lambda)=$ $=\operatorname{det}(A-\lambda E)$.

Zeros of the polynomial $P_{n+1}(\lambda)$ determine all eigenvalues of matrix $A$. Consider the equation

$$
A \vec{y}-\lambda \vec{y}=P_{n+1}(\lambda) \vec{\delta}_{0}, \quad \vec{\delta}_{0}^{T}=(1,0, \ldots, 0)
$$


Assertion 1. For any complex value $\lambda$ there exists a vector $\vec{y}=\vec{y}(\lambda)$, which satisfies the equation (1).

Let $\lambda$ be a fixed complex number. Replace the column $j$ of matrix $(A-\lambda E)$ by the column $\vec{\delta}_{0}^{T}$. By $y_{j-1}(\lambda), j=1,2, \ldots . . n+1$ denote the determinant of the modified matrix. Compose the vector $(\vec{y}(\lambda))^{T}=\left(y_{0}(\lambda), y_{1}(\lambda), \ldots \ldots ., y_{n-1}(\lambda)\right)$. By direct checking we are convinced, that $y_{j-1}(\lambda)$ are polynomials from $\lambda$, and vector $\vec{y}(\lambda)$ satisfies the equation (1) for any complex $\lambda$.

Note that

$$
y_{0}(\lambda)=\operatorname{det}\left[\begin{array}{cccccccc}
a_{1}-\lambda & b_{1} & c_{1} & & & & & \\
b_{1} & a_{2}-\lambda & b_{2} & c_{2} & & & & \\
& c_{1} & a_{3}-\lambda & b_{3} & c_{3} & & & \\
& \ddots & \ddots & \ddots & \ddots & \ddots & & \\
& & \ddots & \ddots & \ddots & \ddots & \ddots & \\
& & & c_{n-4} & b_{n-3} & a_{n-2}-\lambda & b_{n-2} & c_{n-2} \\
& & & & c_{n-3} & b_{n-2} & a_{n-1}-\lambda & b_{n-1} \\
& & & & & c_{n-2} & b_{n-1} & a_{n}-\lambda
\end{array}\right]
$$

and its zeros coincide with the eigenvalues of the truncated matrix $B$ of the dimensionality $n \times n$, where

$$
B=\left[\begin{array}{cccccccc}
a_{1} & b_{1} & c_{1} & & & & & \\
b_{1} & a_{2} & b_{2} & c_{2} & & & & \\
c_{1} & b_{2} & a_{3} & b_{3} & c_{3} & & & \\
& \ddots & \ddots & \ddots & \ddots & \ddots & & \\
& & \ddots & \ddots & \ddots & \ddots & \ddots & \\
& & & c_{n-4} & b_{n-3} & a_{n-2} & b_{n-2} & c_{n-2} \\
& & & & c_{n-3} & b_{n-2} & a_{n-1} & b_{n-1} \\
& & & & & c_{n-2} & b_{n-1} & a_{n}
\end{array}\right] .
$$

The eigenvalues of initial matrix we will number in the order of their growth

$$
\lambda_{0} \leq \lambda_{1} \leq \ldots . \leq \lambda_{n}
$$

Eigenvalues of matrix $B$ analogously number

$$
\mu_{1} \leq \mu_{2} \leq \ldots . \leq \mu_{n}
$$

In the following the zeros of element

$$
y_{1}(\lambda)=\left[\begin{array}{ccccccc}
b_{0} & b_{1} & c_{1} & & & & \\
c_{0} & a_{2}-\lambda & b_{2} & c_{2} & & & \\
& b_{2} & a_{3}-\lambda & b_{3} & c_{2} & & \\
& & \ddots & \ddots & \ddots & & \\
& & & \ddots & \ddots & \ddots & \\
& & & & \ddots & \ddots & \ddots \\
& & & & c_{n-2} & b_{n-1} & a_{n}-\lambda
\end{array}\right]
$$


have important role.

$y_{1}(\lambda)$ is polynomial from $\lambda$ the degree $(n-1)$ with the leading coefficient $\left(b_{0}(-1)^{n-1}\right)$.

By

$$
\left|\eta_{2}\right| \leq\left|\eta_{3}\right| \leq \ldots . \leq\left|\eta_{n}\right|
$$

denote the zero of polynomial $y_{1}(\lambda)$.

We will use the spectrum of the matrix $C$. Matrix $C$ is obtained from the matrix $B$ by the deletion of the first line and first column.

$$
\xi_{2} \leq \xi_{3} \leq \ldots . \leq \xi_{n}
$$

are eigenvalues of matrix $C$ in the order of their growth.

\section{Formulation of the problem of the restoration}

All elements

$a_{0}, a_{1}, \ldots, a_{n}, b_{0}, b_{1}, \ldots, b_{n}, c_{0}, c_{1}, \ldots, c_{n-2} \quad$ of matrix $A$ to restore on the sequences (2), (3), (4), (5).

The number $b_{0}$ is assigned, and one of the numbers of sequence (4) is considered unknown. The algorithm is illustrated below.

\section{Results of the direct problem}

Some properties of the sequences (2), (3), (5).

Lemma 1.The following identity is satisfied for any

$$
\begin{gathered}
\lambda \neq \mu \\
<\vec{y}(\lambda) ; \vec{y}(\mu)>=\frac{P_{n+1}(\lambda) y_{0}(\mu)-P_{n+1}(\mu) y_{0}(\lambda)}{\lambda-\mu} .
\end{gathered}
$$

Consequence 1. The equality

$$
\|\vec{y}(\lambda)\|^{2}=P_{n+1}^{\prime}(\lambda) y_{0}(\lambda)-P_{n+1}(\mu) y_{0}^{\prime}(\lambda)
$$

is fulfill for any $\lambda$.

Proof of the lemma 1.

Accoding to (1) the correspondence

$$
A \vec{y}(\lambda)=\lambda \vec{y}(\lambda)+P_{n+1}(\lambda) \vec{\delta}_{0}
$$

The vector equality

$$
A \vec{y}(\mu)=\mu \vec{y}(\mu)+P_{n+1}(\mu) \vec{\delta}_{0}
$$

is fulfill for any $\mu$.

By scalar multiplication of all members of equality (6) to the vector $\vec{y}(\mu)$, we obtain:

$$
<A \vec{y}(\lambda) ; \vec{y}(\mu)>=\lambda<\vec{y}(\lambda), \vec{y}(\mu)>+P_{n+1}(\lambda) y_{0}(\mu)
$$

Analogously from (7) we obtain

$$
<A \vec{y}(\mu) ; \vec{y}(\lambda)>=\mu<\vec{y}(\mu), \vec{y}(\lambda)>+P_{n+1}(\mu) y_{0}(\lambda)
$$

Subtract equality (9) from (8)and using $A=A^{T}$, we obtain result of lemma 1 .

Consequence 1 follow from lemmal for $\mu \rightarrow \lambda$.

By $\Lambda$ denote the set of complex $\lambda$ for $\|\vec{y}(\lambda)\|>0$.

Theorem 1. The following assertions are fulfill on the set $\Lambda$ :

a) All eigenvalues of matrixes $A, C$ and $B$, which belong to $\Lambda$, simple and real;

b) Eigenvalues of matrixes $A$ and $B, B$ and $C$, which belong to $\Lambda$, do not coincide;

c) the following inequalities fulfill for eigenvalues

$\lambda_{0}<\mu_{1}<\lambda_{1}<\mu_{2}<\ldots .<\mu_{n-1}<\lambda_{n-1}$

Matrixes $A$ and $B$ have the real simple eigenvalues(являются существенно простыми матрицами), and eigenvalues of matrixes $A$ and $B$ interchange.

Proof of theorem 1 .

Let $\lambda_{0} \in \Lambda$ is eigenvalues of matrix $A$, then $P_{n+1}\left(\lambda_{0}\right)=0$. Accoding to consequence 1 we have $0<\|\vec{y}(\lambda)\|^{2}=P_{n+1}^{\prime}(\lambda) y_{0}(\lambda)$. Then the numbers $P_{n+1}^{\prime}\left(\lambda_{0}\right)$ and $y_{0}\left(\lambda_{0}\right)$ are not equal to zero. It means that $P_{n+1}^{\prime}\left(\lambda_{0}\right) \neq 0$, and $\lambda_{0}$-is simple eigenvalue of matrix $B$.

is fulfill for any $\lambda$. 
Assume that $\mu_{0} \in \Lambda$ is eigenvalue of matrix $B$, then $y_{0}\left(\mu_{0}\right)=0$.

Accoding to consequence 1 the following equality is carried out

$$
0<\left\|\vec{y}\left(\mu_{0}\right)\right\|^{2}=-P_{n+1}\left(\mu_{0}\right) y_{0}^{\prime}\left(\mu_{0}\right)
$$

Consequently, the number $P_{n+1}\left(\mu_{0}\right)$ is not equal to zero, thus $\mu_{0}$ cannot be eigenvalue of matrix $A$. From other side it follows from the inequality $y_{0}^{\prime}\left(\mu_{0}\right) \neq 0$, that $\mu_{0}$ is simple eigenvalue of matrix $B$. Assertions prove analogously for matrix $C$.

Observation. If $\lambda_{0} \bar{\in} \Lambda$ then $\vec{y}\left(\lambda_{0}\right)=0$, that is all elements of vector $\vec{y}\left(\lambda_{0}\right)$ are equal to zero. Hence, in particular it follows that $P_{n+1}\left(\lambda_{0}\right)=0$. That is all numbers $P_{n+1}\left(\lambda_{0}\right), \quad y_{0}\left(\lambda_{0}\right)$, $y_{1}\left(\lambda_{0}\right), \ldots, y_{n}\left(\lambda_{0}\right)$ are equal to zero for $\lambda_{0} \bar{\in} \Lambda$.

\section{Results on the problem of the restoration}

In this point we consider that three sequences of the numbers assigned (2), (3), (4), (5). We should find three sequences

$$
\begin{array}{ccc}
a_{0} & a_{1} \ldots, & a_{n} \\
b_{1} & b_{2} \ldots, & b_{n} \\
c_{1} & c_{2} \ldots, & c_{n-1}
\end{array}
$$

Examine the equation

$$
(A-\lambda E) \vec{x}(\lambda)=\vec{\delta}_{0}
$$

By $x_{0}(\lambda), x_{1}(\lambda), \ldots, x_{n}(\lambda)$ denote the elements of vector $\vec{x}(\lambda)$.

For example, for $x_{0}(\lambda)$ the following formula is fulfill

$$
x_{0}(\lambda)=\frac{y_{0}(\lambda)}{P_{n+1}(\lambda)}, \text { if } P_{n+1}(\lambda) \neq 0
$$

Analogously, the equality is correct

$$
x_{1}(\lambda)=-\frac{y_{1}(\lambda)}{P_{n+1}(\lambda)}, \text { if } P_{n+1}(\lambda) \neq 0 .
$$

From the other side, for $|\lambda|>\|A\|$, using a number of Neumann, we obtain the relationship

$$
\begin{gathered}
\vec{x}(\lambda)=(A-\lambda E)^{-1} \vec{\delta}_{0}=-\lambda^{-1}\left(E-\frac{1}{\lambda} A\right)^{-1} \vec{\delta}_{0}= \\
-\frac{1}{\lambda}\left(E+\frac{1}{\lambda} A+\frac{1}{\lambda^{2}} A^{2}+\ldots\right) \vec{\delta}_{0}=-\frac{1}{\lambda} \vec{\delta}_{0}-\frac{1}{\lambda^{2}} A \vec{\delta}_{0}-\frac{1}{\lambda^{3}} A^{2} \vec{\delta}_{0}-\ldots
\end{gathered}
$$

Hence

$$
x_{0}(\lambda)=\left\langle\vec{x}(\lambda), \vec{\delta}_{0}\right\rangle=-\frac{1}{\lambda}-\frac{1}{\lambda^{2}}\left\langle A \vec{\delta}_{0} ; \vec{\delta}_{0}\right\rangle-\frac{1}{\lambda^{3}}\left\langle A^{2} \vec{\delta}_{0} ; \vec{\delta}_{0}\right\rangle-\ldots
$$

Examining the equality (11) more detail for $\left|\lambda_{0}\right| \geq\|A\|=\left|\lambda_{n}\right|$, we obtain 


$$
\begin{aligned}
& x_{0}(\lambda)=-\frac{y_{0}(\lambda)}{P_{n+1}(\lambda)}=\frac{\left(\mu_{1}-\lambda\right)\left(\mu_{2}-\lambda\right) \ldots\left(\mu_{n}-\lambda\right)}{\left(\lambda_{0}-\lambda\right)\left(\lambda_{1}-\lambda\right) \ldots \ldots\left(\lambda_{n}-\lambda\right)}=\frac{y_{0}\left(\lambda_{0}\right)}{P_{n+1}^{\prime}\left(\lambda_{0}\right)\left(\lambda_{0}-\lambda\right)}+ \\
& \frac{y_{0}\left(\lambda_{1}\right)}{P_{n+1}^{\prime}\left(\lambda_{1}\right)\left(\lambda_{1}-\lambda\right)}+\ldots+\frac{y_{0}\left(\lambda_{n}\right)}{P_{n+1}^{\prime}\left(\lambda_{n}\right)\left(\lambda_{n}-\lambda\right)}=\frac{y_{0}(\lambda)}{P_{n+1}^{\prime}(\lambda)}\left\{\frac{1}{\lambda}+\frac{\lambda_{0}}{\lambda^{2}}+\frac{\lambda_{0}{ }^{2}}{\lambda^{3}}+\ldots\right\}+ \\
& +\frac{y_{0}(\lambda)}{P_{n+1}^{\prime}(\lambda)}\left\{\frac{1}{\lambda}+\frac{\lambda_{1}}{\lambda^{2}}+\frac{\lambda_{1}^{2}}{\lambda^{3}}+\ldots\right\}+\ldots .+\frac{y_{0}(\lambda)}{P_{n+1}^{\prime}(\lambda)}\left\{\frac{1}{\lambda}+\frac{\lambda_{n}}{\lambda^{2}}+\frac{\lambda_{n}^{2}}{\lambda^{3}}+\ldots\right\}
\end{aligned}
$$

Comparing (13) и (14), we obtain the infinite system of the relationships

$$
\left\langle A^{k} \vec{\delta}_{0} ; \vec{\delta}_{0}\right\rangle=-\sum_{0}^{n} \lambda_{j}^{k} \frac{y_{0}\left(\lambda_{j}\right)}{P_{n+1}^{\prime}\left(\lambda_{j}\right)}
$$

for $\mathrm{k}=1,2, .$. Analogously from (12) we obtain the infinite system of the relationships

$$
\left\langle A^{k} \vec{\delta}_{0} ; \vec{\delta}_{1}\right\rangle=-\sum_{0}^{n} \lambda_{j}^{k} \frac{y_{1}\left(\lambda_{j}\right)}{P_{n+1}^{\prime}\left(\lambda_{j}\right)}
$$

for $\mathrm{k}=1,2, .$. The same system of equations follows for the matrix elements $B$

$$
\left\langle B^{k} \vec{\delta}_{0} ; \vec{\delta}_{0}\right\rangle=-\sum_{1}^{n} \mu_{j}^{k} \frac{\operatorname{det}\left(C-\mu_{j} E\right)}{y_{0}^{\prime}\left(\mu_{j}\right)} .
$$

Systems (15), (16), (17) are infinite system for determining the elements of the matrix $A$ from the known right sides. Let $\vec{\delta}_{k}$ is the vector with the zero components, besides $(k+1)-$, which is equal to one. Produce some calculations. Note

$$
\begin{aligned}
& A \vec{\delta}_{0}=a_{0} \vec{\delta}_{0}+b_{0} \vec{\delta}_{1}+c_{0} \vec{\delta}_{2}, \\
& A \vec{\delta}_{1}=b_{0} \vec{\delta}_{0}+a_{1} \vec{\delta}_{1}+b_{1} \vec{\delta}_{2}+c_{1} \vec{\delta}_{3}, \\
& A \vec{\delta}_{k}=c_{k-2} \vec{\delta}_{k-2}+b_{k-1} \vec{\delta}_{k-1}+a_{k} \vec{\delta}_{k}+b_{k} \vec{\delta}_{k+1}+c_{k} \vec{\delta}_{k+2}, \\
& k=2,3, \ldots n-2 \\
& A \vec{\delta}_{n-1}=c_{n-3} \vec{\delta}_{n-3}+b_{n-2} \vec{\delta}_{n-2}+a_{n-1} \vec{\delta}_{n-1}+b_{n-1} \vec{\delta}_{n}, \\
& A \vec{\delta}_{n}=c_{n-2} \vec{\delta}_{n-2}+b_{n-1} \vec{\delta}_{n-1}+a_{n} \vec{\delta}_{n} .
\end{aligned}
$$

By the induction it is easy to prove, that

$$
A^{k} \vec{\delta}_{0}=d_{0}^{(k)} \vec{\delta}_{0}+d_{1}^{(k)} \vec{\delta}_{1}+\ldots+d_{2 k}^{(k)} \vec{\delta}_{2 k}
$$

Following recurrence formulas are true for the coefficients $d_{j}^{(k+1)}$ :

$$
\left\{\begin{array}{l}
d_{0}^{(k+1)}=a_{0} d_{0}^{(k)}+b_{0} d_{1}^{(k)}+c_{0} d_{2}^{(k)} \\
d_{1}^{(k+1)}=b_{0} d_{0}^{(k)}+a_{1} d_{1}^{(k)}+b_{1} d_{2}^{(k)}+c_{1} d_{3}^{(k)} \\
d_{2}^{(k+1)}=c_{0} d_{0}^{(k)}+b_{1} d_{1}^{(k)}+a_{2} d_{2}^{(k)}+b_{2} d_{3}^{(k)}+c_{2} d_{4}^{(k)} \\
d_{j}^{(k+1)}=c_{j-2} d_{j-2}^{(k)}+b_{j-1} d_{j-1}^{(k)}+a_{j} d_{j}^{(k)}+b_{j} d_{j+1}^{(k)}+c_{j} d_{j+2}^{(k)}
\end{array}\right.
$$

and 


$$
d_{0}^{(1)}=a_{0} ; d_{1}^{(1)}=b_{0} ; d_{2}^{(1)}=c_{0} .
$$

Here, subsequently we consider that the numbers $a_{j}, b_{j-1}, c_{j-2}$ are equal zero for $j>n$. Hence it is apparent that coefficients $d_{j}^{(k)}$ depend only on the row elements of matrix $A$ with the numbers (0), (1), (2), (3),...., $(2 k-3),(2 k-2)$. Formulate this result in the form of lemma.

Lemma 1. Let $k$ is fixed natural number. For $j=0,1, \ldots, 2 k-1$ the numbers $d_{j}^{(k+1)}$ depends only on the collection $\left\{a_{0}, b_{0}, c_{0}, a_{1}, b_{1}, c_{1}, \ldots, a_{2 k-1}, b_{2 k-1}, c_{2 k-1}\right\}$. The numbers $d_{2 k}^{(k+1)}, d_{2 k+1}^{(k+1)}, d_{2 k+2}^{(k+1)}$ have representation

and

$$
\begin{aligned}
& c_{0}^{(k+1)}=a_{0} c_{0}^{(k)}+b_{0} c_{1}^{(k)}+c_{0} c_{2}^{(k)}, \\
& c_{1}^{(k+1)}=b_{0} c_{0}^{(k)}+a_{1} c_{1}^{(k)}+b_{1} c_{2}^{(k)}+c_{1} c_{2}^{(k)}, \\
& c_{j}^{(k+1)}=c_{j-2} c_{j-2}^{(k)}+b_{j-1} c_{j-1}^{(k)}+a_{j} c_{j-1}^{(k)}+b_{j} c_{j-1}^{(k)}+c_{j} c_{j+2}^{(k)}
\end{aligned}
$$

where $f_{2 k}, f_{2 k+1}$ depends only on the collection $\left\{a_{0}, b_{0}, c_{0}, a_{1}, b_{1}, c_{1}, \ldots, a_{2 k-1}, b_{2 k-1}, c_{2 k-1}\right\}$.

Analogously we obtain following representation

$$
A^{k} \vec{\delta}_{1}=c_{0}^{(k)} \vec{\delta}_{0}+c_{1}^{(k)} \vec{\delta}_{1}+\ldots+c_{2 k+1}^{(k)} \vec{\delta}_{2 k+1},
$$

For coefficient $c_{j}^{(k)}$ the following recurrence formulas are true.

$$
c_{0}^{(1)}=b_{0} ; c_{1}^{(1)}=a_{0} ; c_{2}^{(1)}=b_{0} ; c_{3}^{(1)}=c_{1} .
$$

Note that the coefficients $c_{j}^{(k)}$ depend only on the row elements of matrix $A$ with the numbers $(0),(1),(2), \ldots .(2 k-2),(2 k-1)$.

Formulate this result in the form of lemma.

Lemma 2. Let $k$ is fixed natural number. For $j=0,1, \ldots, 2 k$ the numbers $c_{j}^{(k+1)}$ depend only on the collection

$\left\{a_{0}, b_{0}, c_{0}, a_{1}, b_{1}, c_{1}, \ldots, a_{2 k}, b_{2 k}, c_{2 k}\right\}$.

The numbers $c_{2 k+3}^{(k+1)}, c_{2 k+1}^{(k+1)}, c_{2 k+2}^{(k+1)}$ have representation

$$
c_{2 k+1}^{(k+1)}=a_{2 k+1} c_{2 k+1}^{(k)}+r_{2 k+1},
$$

$$
\begin{gathered}
c_{2 k+2}^{(k+1)}=b_{2 k+1} c_{2 k+1}^{(k)}+r_{2 k+2}, \\
c_{2 k+3}^{(k+1)}=c_{2 k+1} c_{2 k+1}^{(k)}
\end{gathered}
$$

where $r_{2 k+2}, r_{2 k+1}$ depend only on the collection $\left\{a_{0}, b_{0}, c_{0}, a_{1}, b_{1}, c_{1}, \ldots, a_{2 k}, b_{2 k}, c_{2 k}\right\}$.

Lemma 3. For any natural $k$ the following representation is true

$$
\left\langle A^{2 k+1} \vec{\delta}_{0} ; \vec{\delta}_{0}\right\rangle=a_{2 k} d_{2 k}^{(k)} d_{2 k}^{(k)}+g_{2 k-1}
$$

where $g_{2 k-1}$ depend only on the collection $\left\{a_{0}, b_{0}, c_{0}, a_{1}, b_{1}, c_{1}, \ldots, a_{2 k-1}, b_{2 k-1}, c_{2 k-1}\right\}$.

For the proof of lemma 3 examine the scalar product 


$$
\begin{aligned}
& \left\langle A^{2 k+1} \vec{\delta}_{0} ; \vec{\delta}_{0}\right\rangle=\left\langle A^{k} \vec{\delta}_{0} ; A^{k+1} \vec{\delta}_{0}\right\rangle=\left\langle\sum_{j=0}^{2 k} d_{j}^{(k)} \vec{\delta}_{j} ; \sum_{i=0}^{2 k+2} d_{i}^{(k+1)} \vec{\delta}_{i}\right\rangle=\sum_{j=0}^{2 k} d_{j}^{(k)} d_{j}^{(k+1)}= \\
& =d_{2 k}^{(k)} d_{2 k}^{(k+1)}+\sum_{j=0}^{2 k-1} d_{j}^{(k)} d_{j}^{(k+1)}=d_{2 k}^{(k)}\left(c_{2 k-2} d_{2 k-2}^{(k)}+b_{2 k-1} d_{2 k-1}^{(k)}+a_{2 k} d_{2 k}^{(k)}\right)+\sum_{j=0}^{2 k-1} d_{j}^{(k)} d_{j}^{(k+1)}= \\
& =\left(a_{2 k} d_{2 k}^{(k)} d_{2 k}^{(k)}\right)+c_{2 k-2} d_{2 k-2}^{(k)} d_{2 k}^{(k)}+b_{2 k-1} d_{2 k-1}^{(k)} d_{2 k}^{(k)}+\sum_{j=0}^{2 k-1} d_{j}^{(k)} d_{j}^{(k+1)}
\end{aligned}
$$

Note that the components which are out of round brackets depend only from the first $(2 k-1)$ lines of matrix $A$. From (18)and according to lemma 1 , follows the assertion of the lemma 3 .

Lemma 4. For any natural $\mathrm{k}$ following representation is true

$$
\left\langle A^{2 k+2} \vec{\delta}_{0} ; \vec{\delta}_{0}\right\rangle=\left(a_{2 k}^{2}+b_{2 k}^{2}+c_{2 k}^{2}\right) d_{2 k}^{(k)} d_{2 k}^{(k)}+h_{2 k-1}
$$
where number $h_{2 k-1}$ depend only on the collection $\left\{a_{0}, b_{0}, c_{0}, a_{1}, b_{1}, c_{1}, \ldots, a_{2 k-1}, b_{2 k-1}, c_{2 k-1}\right\}$.

For the proof of lemma 4 examine the scalar product

$$
\begin{gathered}
\left\langle A^{2 k+2} \vec{\delta}_{0} ; \vec{\delta}_{0}\right\rangle=\left\langle A^{k+1} \vec{\delta}_{0} ; A^{k+1} \vec{\delta}_{0}\right\rangle=\left\langle\sum_{j=0}^{2 k+2} d_{j}^{(k+1)} \vec{\delta}_{j} ; \sum_{i=0}^{2 k+2} d_{i}^{(k+1)} \vec{\delta}_{i}\right\rangle=\sum_{j=0}^{2 k+2} d_{j}^{(k+1)} d_{j}^{(k+1)}= \\
=d_{2 k+2}^{(k+1)} d_{2 k+2}^{(k+1)}+d_{2 k+1}^{(k+1)} d_{2 k+1}^{(k+1)}+d_{2 k}^{(k+1)} d_{2 k}^{(k+1)}+\sum_{j=0}^{2 k-1} d_{j}^{(k+1)} d_{j}^{(k+1)}
\end{gathered}
$$

According to lemma 1 follows the assertion of the lemma 3.

Lemma 5. For any natural $k$ following representation is true

$\left\langle A^{2 k} \vec{\delta}_{0} ; \vec{\delta}_{1}\right\rangle=b_{2 k-1} d_{2 k}^{(k)} c_{2 k-1}^{(k-1)}+a_{2 k-1} d_{2 k-1}^{(k)} c_{2 k-1}^{(k-1)}+h_{2 k-2}$

where number $h_{2 k-2}$ depend only on the collection $\left\{a_{0}, b_{0}, c_{0}, a_{1}, b_{1}, c_{1}, \ldots, a_{2 k-2}, b_{2 k-2}, c_{2 k-2}\right\}$.

For the proof of lemma 5 examine the scalar product

$$
\begin{gathered}
\left\langle A^{2 k} \vec{\delta}_{0} ; \vec{\delta}_{1}\right\rangle=\left\langle A^{k} \vec{\delta}_{0} ; A^{k} \vec{\delta}_{1}\right\rangle=\left\langle\sum_{j=0}^{2 k} d_{j}^{(k)} \vec{\delta}_{j} ; \sum_{i=0}^{2 k} c_{i}^{(k)} \vec{\delta}_{i}\right\rangle=\sum_{j=0}^{2 k} d_{j}^{(k)} c_{j}^{(k)}= \\
=d_{2 k}^{(k)} c_{2 k}^{(k)}+d_{2 k-1}^{(k)} c_{2 k-1}^{(k)}+\sum_{j=0}^{2 k-2} d_{j}^{(k)} c_{j}^{(k)}
\end{gathered}
$$

According to lemma 2 we obtain the equality

$$
\begin{gathered}
\left\langle A^{2 k+1} \vec{\delta}_{0} ; \vec{\delta}_{1}\right\rangle=b_{2 k} d_{2 k}^{(k)} c_{2 k-1}^{(k-1)} c_{2 k-1}+ \\
+a_{2 k} d_{2 k}^{(k)} c_{2 k-1}^{(k-1)} b_{2 k-1}+p_{2 k-2}
\end{gathered}
$$$$
\left\langle A^{2 k} \vec{\delta}_{0} ; \vec{\delta}_{1}\right\rangle=d_{2 k}^{(k)} c_{2 k}^{(k)}+d_{2 k-1}^{(k)} c_{2 k-1}^{(k)}+\sum_{j=0}^{2 k-2} d_{j}^{(k)} c_{j}^{(k)}=
$$$$
=d_{2 k}^{(k)} b_{2 k-1} c_{2 k-1}^{(k-1)}+d_{2 k-1}^{(k)} a_{2 k-1} c_{2 k-1}^{(k-1)}+h_{2 k-2}
$$

The assertion of lemma 5 follows from the last formula and the lemmas 1 and 2 .

Lemma 6. For any natural $k$ following represenwhere number $p_{2 k-2}$ depend only on the collection $\left\{a_{0}, b_{0}, c_{0}, a_{1}, b_{1}, c_{1}, \ldots, a_{2 k-2}, b_{2 k-2}, c_{2 k-2}\right\}$.

For the proof of lemma 6 examine the scalar product tation is true 


$$
\begin{gathered}
\left\langle A^{2 k+1} \vec{\delta}_{0} ; \vec{\delta}_{1}\right\rangle=\left\langle A^{k+1} \vec{\delta}_{0} ; A^{k} \vec{\delta}_{1}\right\rangle=\left\langle\sum_{j=0}^{2 k+2} d_{j}^{(k)} \vec{\delta}_{j} ; \sum_{i=0}^{2 k+1} c_{i}^{(k)} \vec{\delta}_{i}\right\rangle=\sum_{j=0}^{2 k+1} d_{j}^{(k+1)} c_{j}^{(k)}= \\
=d_{2 k+1}^{(k+1)} c_{2 k+1}^{(k)}+d_{2 k}^{(k+1)} c_{2 k}^{(k)}+\sum_{j=0}^{2 k-1} d_{j}^{(k+1)} c_{j}^{(k)}
\end{gathered}
$$

According to lemma 1 we obtain the equality

$$
\begin{gathered}
\left\langle A^{2 k+1} \vec{\delta}_{0} ; \vec{\delta}_{1}\right\rangle=\left(b_{2 k} d_{2 k}^{(k)}+f_{2 k+1}\right) c_{2 k+1}^{(k)}+\left(a_{2 k} d_{2 k}^{(k)}+f_{2 k}\right) c_{2 k}^{(k)}+\sum_{j=0}^{2 k-1} d_{j}^{(k+1)} c_{j}^{(k)}= \\
=\left(b_{2 k} d_{2 k}^{(k)} c_{2 k+1}^{(k)}+a_{2 k} d_{2 k}^{(k)} c_{2 k}^{(k)}\right)+p_{2 k-2}
\end{gathered}
$$

Hence follows the assertion of the lemma 6, according to lemma 2.

The following two lemmas relate to the ma$\operatorname{trix} B$, which is analogous to the matrix $A$. Therefore its properties are analogous to the properties of the matrix $A$.

Lemma 7. For any natural $k$ following representation is true

$$
\left\langle B^{2 k+1} \vec{\delta}_{0} ; \vec{\delta}_{0}\right\rangle=a_{2 k+1} \widetilde{d}_{2 k}^{(k)} \widetilde{d}_{2 k}^{(k)}+\widetilde{g}_{2 k-1},
$$

where numbers $\widetilde{g}_{2 k-1}, \widetilde{d}_{2 k}^{(k)}, \widetilde{d}_{2 k}^{(k)}$ depend only on the collection

$$
\left\{a_{1}, b_{1}, c_{1}, a_{2}, b_{2}, c_{2}, \ldots, a_{2 k}, b_{2 k}, c_{2 k}\right\} \text {. }
$$

Lemma 8. For any natural $k$ following representation is true

$$
\left\langle B^{2 k+2} \vec{\delta}_{0} ; \vec{\delta}_{0}\right\rangle=\left(a_{2 k+1}^{2}+b_{2 k+1}^{2}+c_{2 k+1}^{2}\right) \widetilde{d}_{2 k}^{(k)} \widetilde{d}_{2 k}^{(k)}+\widetilde{q}_{2 k-1}
$$

where numbers $\widetilde{q}_{2 k-1}, \widetilde{d}_{2 k}^{(k)}, \widetilde{d}_{2 k}^{(k)}$ depend only on the collection

$$
\left\{a_{1}, b_{1}, c_{1}, a_{2}, b_{2}, c_{2}, \ldots, a_{2 k}, b_{2 k}, c_{2 k}\right\} \text {. }
$$

\section{Conclusion}

Algorithm of the restoration of matrix elements $A$.

1. Let the elements with the numbers $\left\{a_{0}, b_{0}, c_{0}, a_{1}, b_{1}, c_{1}, \ldots, a_{2 k-1}, b_{2 k-1}, c_{2 k-1}\right\}$ are founded already.
2. The element $\left\{a_{2 k}\right\}$ can be found from the lemma 3. It is determined (unequivocal) unambiguously, since $d_{2 k}^{(k)}=c_{2 k-2} c_{2 k-3} \cdots c_{0}>0$

3. The element $\left\{b_{2 k}\right\}$ can be found from the lemma 6. It is determined (unequivocal) unambiguously, since $d_{2 k}^{(k)}, c_{2 k-1}^{(k-1)}, c_{2 k-1}>0$.

4. The element $\left\{c_{2 k}\right\}$ can be found from the lemma 4. It is determined (unequivocal) unambiguously, since $d_{2 k}^{(k)}>0$.

5. The element $\left\{a_{2 k+1}\right\}$ can be found from the lemma 7. It is determined (unequivocal) unambiguously, since $\tilde{d}_{2 k}^{(k)}>0$.

6. The element $\left\{b_{2 k+1}\right\}$ can be found from the lemma 5. It is determined (unequivocal) unambiguously, since $d_{2 k+2}^{(k+1)}, c_{2 k+1}^{(k)},>0$.

7. The element $\left\{c_{2 k+1}\right\}$ can be found from the lemma 8. It is determined (unequivocal) unambiguously, since $\widetilde{d}_{2 k}^{(k)}>0$.

Thus, all elements of initial matrix are restored. Formulate the basic result of work.

Theorem 2. If sequences (1), (2), (4) are eigenvalues of matrixes $A, B, C$, and sequence (3) are zeros of polynomial $y_{1}(\lambda)$, then matrix elements are restored unambiguously on the indicated sequences. The algorithm of restoration is given. 


\section{References}

1. Hochctadt H. On the construction of a Jacobi matrix from spectral date. Linear Algebra and its Applications, 8, 5(1974). - P.435 - 446.

2. Golinskij L.B., Kudryavtsev M. A. Return spectral problems for one class of the five-diagonal unitary matrixes // Dokl. of the Russian Academy of Sciences. 2008, - V.423. - № 1. - P. 11-18.

3. Godunov S.K., Kostin V.I., Mitchenko A.D. Vychislenie sobstvennogo vektora simmetricheskoj trehdiagonal'noj matricy // Sib. mat. zhurnal, 1985. - T. 26. - № 5. - P.71-85.

4. Godunov S.K. Sovremennye aspekty linejnoj algebry,--Novosibirsk: Nauchnaja kniga, 1997,- P.390.

5. Voevodin V.V. Chislennye metody algebry. Teorija i algorifmy. - M.: Nauka, 1966. - $235 \mathrm{~s}$.

6. Uilkinson Dzh. X. Algebraicheskaja problema sobstvennyh znachenij. - M.: Nauka, 1970. $108 \mathrm{~s}$.

7. Danilina N.I., Dubrovskaja N.S., Kvasha O.P., Smirnov G.L., Fekansov G.I. Chislennye metody. - M.: Vysshaja shkola, 1976. - P.368.
8. Matematicheskij jenciklopedicheskij slovar, - M.: Sovetskaja jenciklopedija, 1988. - P.847.

9. Bahvalov N.S. Chislennye metody. - M.: Nauka, 1973. - P.632.

10. Horn R. Dzhonson Ch. Matrichnyj analiz, M.: Izd-vo «Mir», 1988. - P.206.

11. Ikramov H.D. Nesimmetrichnaja problema sobstvennyh znacheni. - M.: Nauka, 1991. - P.106.

12. Ikramov H.D. Chislennye metody linejnoj algebry. (Reshenie linejnyh uravnenij). - M.: Znanie, 1987. - P.241.

13. Markus M., Mink H. Obzor po teori matric i matrichnyh neravenstv. - M.: Nauka, 1972. - P. 34.

14. Ikramov H.D. Chislennye metody dlja simmetrichnyh linejnyh sistem. - M.: Nauka, 1988. P.102.

15. Ikramov H.D. Vychislitel'nye metody linejnoj algebry. (Reshenie bol'shih razrezhennyh sistem uravnenij prjamymi metodami), - M.: Znanie. 1989. - P.84.

16. Parlett B. Simmetrichnaja problema sobstvennyh znachenij. - M.: Mir, 1983. - P.76. 